\title{
Conexión, conectividad y socialidad online. Un acercamiento a la evolución de las redes sociales en la web 2.0
}

\author{
Connection, connectivity and online sociability. An aproach to the \\ evolution of social media in a 2.0 web
}

Constanza María Ferrario ferrario.constanza@gmail.com

http://orcid.org/0000-0001-7013-4897

Grupo de investigación sobre familia, género y subjetividades; Facultad de Humanidades; Universidad Nacional de Mar del Plata (Argentina)

\section{Resumen}

En esta reseña se exponen los principales conceptos, análisis y aportes referidos al estudio de redes sociales y socialidad online desarrollados por José Van Dijck en La cultura de la conectividad. Una historia critica de las redes sociales. Se retoma el análisis de cinco plataformas virtuales (Facebook, Twitter, Flickr, YouTube y Wikipedia) y se focaliza en el pasaje de una socialidad aparentemente desinteresada y participativa a una socialización basada en la obtención y venta de datos privados. Se analiza la tensión constante entre conexión y conectividad al mismo tiempo que se desarrolla la importancia del verbo compartir. Se relacionan los aportes teóricos de la autora con los enfoques centrados en el rol celebratorio de los usuarios, las teorías alusivas al concepto de poder en Foucault y las posturas economicistas referidas a los flujos de capital. Hacia el final, se enfatiza en la naturalización del uso de medios conectivos, en la importancia actual de las redes sociales para el desarrollo de 
la sociabilidad y en las dificultades que genera para los usuarios intentar salir de esas plataformas online.

Palabras clave: Conexión; conectividad; socialidad online; redes sociales; José Van Dijck.

\begin{abstract}
In this review are exposed the main concepts, analysis and inputs referred to the study of the social networks and online sociality developed by Jose Van Dijck in The Culture of connectivity. It brings back the analysis of 5 virtual platforms (Facebook, Twitter, Flickr, YouTube and Wikipedia) and makes focus in the way through from an apparent needless and participative sociality to a socialization based on getting and selling private information. It analyzes the constant tension between connection and connectivity, and at the same time, it develops the importance of the verb share. It relates the theoretical inputs from the author with the focus centered on the celebratory role of the users, the allusive theories to the power concept on Foucault and the economic postures referred to the capital flows. To the end, it emphasizes the naturalized use of connective media, the recent importance of social networks for the development of sociability and the difficulties that this creates for users that try to leave these platforms.
\end{abstract}

Keywords: Connection; connectivity; online sociability; social networks; Jose Van Dijck.

En un contexto donde las nuevas tecnologías y sus modos de uso aparecen de manera naturalizada y las redes sociales se vuelven la condición necesaria para la interacción social, Van Dijck brinda a través de su estudio, un análisis exhaustivo, detallado, riguroso y por sobre todo critico de la evolución que han tenido las redes sociales en los últimos diez años.

Para la autora lo que atrajo a la mayoría de los usuarios a la web 2.0 fue la necesidad de la conexión, poder relacionarse con otros, construir comunidades, fomentar la colaboración, la creatividad y desarrollar una cultura participativa que orientara a volver la red un ámbito social. Pero las empresas y los dueños de las plataformas, al ver la explosión en la cantidad de usuarios y la potencialidad de generar dinero, se alejaron del fomento a la formación de comunidades para acercarse a la búsqueda de los datos personales de los usuarios. Así es que para Van Dijck la conectividad se fue convirtiendo en un recurso valioso.La autora busca a lo largo del libro analizar esta tensión entre la "conexión" y la "conectividad". Es decir, examinar 
el pasaje producido en el lapso de una década, de una comunicación en red basada en una socialización aparentemente desinteresada y una cultura participativa, a una socialidad moldeada por las redes sociales que buscan la rentabilidad, en otras palabras, a una cultura de la conectividad.

La autora analiza la historia de cinco de las plataformas más relevantes en la última década: Facebook (2004), Twitter (2006), Flickr (2004), YouTube (2005) y Wikipedia (2001). El estudio de dichas redes se realiza en términos ecológicos, esto quiere decir que Van Dijck si bien plantea la necesidad de abordarlas de manera individual, ya que algunas adquieren más relevancia que otras, no se detiene meramente en cada una de ellas, sino que las posiciona dentro de un sistema mayor, donde estas redes o microsistemas están interconectados. La totalidad de estas plataformas es definida como "ecosistema de medios conectivos". Estas redes se van modificando en relación a las actividades de los usuarios, como así también a las necesidades o los intereses de los propietarios y a la relación que establecen con otras plataformas; esto es lo que para Van Dijck le brinda al ecosistema un carácter cambiante.

Si bien en numerosas entrevistas y fragmentos de su libro la autora da cuenta de dicho carácter vertiginoso y cambiante, el panorama no parece ser muy positivo. Para ella las redes sociales atraparon a los usuarios. Una vez que dieron el clic y se convirtieron en miembros de alguna de dichas plataformas, será muy difícil poder salir. Como especifica la autora, las redes sociales se valen de algunos comportamientos de la vida real para moldear y manipular las relaciones sociales online. "Compartir", "hacer amigos", "seguir", dar "me gusta" son significados que como se demuestra, se volvieron dominantes. Haciendo uso de los aportes de Foucault, la autora evidencia como estos elementos se convirtieron en una norma social con un gran poder que articula y orienta las interacciones tanto fuera como dentro de la red. Así, los medios conectivos se volvieron casi sinónimos de socialidad, lo que hace que cada vez que alguien intente salir de ellos se enfrente a la presión social de sus amigos, colegas y familiares para que no lo haga.

El libro presenta una manera de percibir la red social donde los usuarios son importantes pero las tecnologías también lo son. Alejándose de aquellas perspectivas que se centraban solamente en celebrarla participación y el rol protagónico de los usuarios en las redes, Van Dijckplantea que la lógica de la conectividad avanza sobre la lógica de la conexión lo que implica que elementos como los protocolos, los meta-datos, los algoritmos, las interfaces, etc., deben volverse factores activos en el análisis de las redes sociales. También se destaca la centralidad del análisis de los contenidos y los usuarios y es por ello que se dedican algunas partes del libro al estudio de los usuarios más críticos, es decir, aquellos que responden a los mecanismos de monetización y a los cambios en la privacidad de cada una de las plataformas 
en las que participan. Si bien esto hace que se reconozca su importancia, también se destaca la forma en la cual las tecnologías son puestas al servicio del capital y no de los usuarios, lo que reduciría de la capacidad de agencia de estos últimos.

Por otro lado, desde la mirada de la economía política, Van Dijck se interesa por los flujos de capital y por las relaciones que se establecen entre los marcos políticos, legales y económicos profundizando en los regímenes de propiedad, de gobierno y en las formas que adquieren los negocios en cada una de estas redes sociales. En este sentido es que la autora se interesa por los límites entre lo público y lo privado y el control de la información que comparten los usuarios en relación con las plataformas y el gobierno. Los valores referidos a la privacidad de los contenidos, la búsqueda de un espacio público libre de la contaminación de los intereses comerciales y la conciencia de saber quién está controlando ese espacio eran los que los usuarios que buscaban la conexión en la web 2.0 daban por sentado, pero que con el avance de la conectividad empiezan a desaparecer.

El verbo que para la autora moviliza a todas estas redes sociales es el de "compartir". Popularizado inicialmente por Facebook y luego establecido como el principio para la interacción, este concepto da cuenta de la distribución de la información por parte de los usuarios y también de la filtración de esa información hacia terceros. La acción de compartir es central en la obra de la autora, ya que le permite realizar el análisis de cada una de las redes sociales y del ecosistema general desde la perspectiva del capital y desde la de los usuarios. De esta forma, se analiza qué es aquello que impulsa a los usuarios a compartir información con otros a través de determinadas interfaces y también se visibiliza cómo los propietarios de las plataformas comparten los datos de los usuarios con terceros para obtener ganancias.

La conectividad aparece organizada a través de botones como el "me gusta" en Facebook o "el botón T" de Twitter y son algoritmos, cada vez mas compatibles e intercambiables que codifican los gustos y preferencias de los usuarios y son capaces de brindar una enorme cantidad de datos agregados. A lo largo de la obra, la autora se refiere a estos mecanismos invisibles a través de los cuales las conexiones y acciones en cada una de las plataformas en las que los usuarios interactúan se convierten en datos que se vuelven mercancías, para ser vendidas a terceros. Al mismo tiempo se reconoce cómo la producción de estos datos sociales son utilizados por las plataformas para incidir en los perfiles individuales y colectivos de los usuarios. Así, se personalizan las búsquedas y se vuelve visible a los usuarios solo lo que las mismas plataformas creen apropiado. En el mismo sentido, se analiza esta lógica a través de los rankings de popularidad de las plataformas; allí, la cantidad de seguidores en Twitter, los amigos en Facebook, o las calificaciones en YouTube determinan qué personas son más valiosas que otras dentro de la socialidad online. Esto le permite a Van Dijck explicar cómo la 
popularidad también se codifica y se vuelve no solo cuantificable sino manipulable. Cada una de estas plataformas sigue una lógica particular, son capaces de convertirse en una empresa de datos donde bajo el disfraz de la transparencia y la conexión para los usuarios producen conectividad.

En resumidas palabras La cultura de la conectividad brinda las herramientas necesarias para indagar en los mecanismos invisibles que los medios conectivos, bajo la lógica del bien común y la trasparencia, ocultan. Van Dijck a través del análisis de la tensión entre "conexión" y "conectividad" y la importancia que adquiere el verbo "compartir" en estas redes sociales, es capaz de realizar un análisis detallado y sobre todo critico de la evolución y las transformaciones que estas plataformas han tenido en la última década.Parecería que ya no hay salida: la socialidad y comunicación en red no se dirige hacia la conexión que buscaban los usuarios de la web 2.0 sino que se acerca cada vez más a convertirse en una cultura de la conectividad. Tal como se demuestra en este libro, la última década ha hecho que las redes sociales se conviertan en la condición necesaria para la interacción social y que el uso de las nuevas tecnologías aparezca de manera naturalizada en la vida cotidiana. En ese contexto, los actuales y futuros usuarios ¿aceptarían sumarse a una red que no tuviera esas características? Sin duda alguna Van Dijck en la Cultura de la Conectividad ofrece las herramientas necesarias para comenzar a pensarlo.

\section{Bibliografía}

Van Dijck, J. (2016). La cultura de la conectividad: una historia crítica de las redes sociales. Buenos Aires: Siglo Veintiuno Editores. 